\title{
Carrier-Density-Dependent Lattice Stability in InSb
}

\author{
P. B. Hillyard, ${ }^{1,2}$ K. J. Gaffney, ${ }^{2,3, *}$ A. M. Lindenberg, ${ }^{2,3}$ S. Engemann, ${ }^{2,3}$ R. A. Akre, ${ }^{4}$ J. Arthur, ${ }^{3}$ C. Blome, ${ }^{5}$ \\ P. H. Bucksbaum, ${ }^{2,3,6}$ A. L. Cavalieri, ${ }^{6,7}$ A. Deb, ${ }^{2,3}$ R. W. Falcone, ${ }^{8}$ D. M. Fritz, ${ }^{2,6}$ P. H. Fuoss, ${ }^{9}$ J. Hajdu, ${ }^{10}$ P. Krejcik, ${ }^{4}$ \\ J. Larsson, ${ }^{11}$ S. H. Lee, ${ }^{6,12}$ D. A. Meyer, ${ }^{1,2}$ R. Pahl, ${ }^{13}$ D. A. Reis, ${ }^{2,6}$ J. Rudati, ${ }^{14}$ D. P. Siddons, ${ }^{15}$ K. Sokolowski-Tinten, ${ }^{16}$ \\ D. von der Linde, ${ }^{16}$ and J. B. Hastings ${ }^{3}$ \\ ${ }^{1}$ Department of Chemistry, Stanford University, Stanford, California 94305, USA \\ ${ }^{2}$ PULSE Center, Stanford Linear Accelerator Center, Menlo Park, California 94025, USA \\ ${ }^{3}$ Stanford Synchrotron Radiation Laboratory/SLAC, Menlo Park, California 94025, USA \\ ${ }^{4}$ Stanford Linear Accelerator Center, Menlo Park, California, 94025, USA \\ ${ }^{5}$ Deutsches Elektronen-Synchrotron DESY, Notkestrasse 85, 22607 Hamburg, Germany \\ ${ }^{6}$ FOCUS Center, Department of Physics and Applied Physics Program, University of Michigan, Ann Arbor, Michigan 48109, USA \\ ${ }^{7}$ Max-Planck-Institute of Quantum Optics, Hans-Kopfermann-Strasse 1, D-85748, Garching, Germany \\ ${ }^{8}$ Department of Physics, University of California, Berkeley, California 94720, USA \\ ${ }^{9}$ Materials Science Division, Argonne National Laboratory, Argonne, Illinois 60439, USA \\ ${ }^{10}$ Department of Cell and Molecular Biology, Biomedical Centre, Uppsala University, SE-75124 Uppsala, Sweden \\ ${ }^{11}$ Department of Physics, Lund Institute of Technology, P.O. Box 118, S-22100 Lund, Sweden \\ ${ }^{12}$ Length/Time Metrology Group, Korea Institute of Standards and Science, Daejeon 305-600, Republic of Korea \\ ${ }^{13}$ Consortium for Advanced Radiation Sources, The University of Chicago, Chicago, Illinois 60637, USA \\ ${ }^{14}$ Advanced Photon Source, Argonne National Laboratory, Argonne, Illinois 60439, USA \\ ${ }^{15}$ National Synchrotron Light Source, Brookhaven National Laboratory, Upton, New York 11973, USA \\ ${ }^{16}$ Institut für Experimentelle Physik, Universität Duisberg-Essen, D-45117 Essen, Germany
}

(Received 22 October 2006; published 21 March 2007)

\begin{abstract}
The ultrafast decay of the x-ray diffraction intensity following laser excitation of an InSb crystal has been utilized to observe carrier dependent changes in the potential energy surface. For the first time, an abrupt carrier dependent onset for potential energy surface softening and the appearance of accelerated atomic disordering for a very high average carrier density have been observed. Inertial dynamics dominate the early stages of crystal disordering for a wide range of carrier densities between the onset of crystal softening and the appearance of accelerated atomic disordering.
\end{abstract}

DOI: $10.1103 /$ PhysRevLett.98.125501

First-order phase transitions and chemical reactions require crossing a transition state on the potential energy surface (PES). Characterizing the topography of the energy landscape in the vicinity of the transition state represents the key step to understanding the pathway followed during a chemical reaction or first-order phase transition. The experimental and theoretical characterization of these far from equilibrium regions of the PES has proven to be very difficult because of the vanishingly short time spent near the transition state and the multitude of degrees of freedom that influence chemical and physical transformations in the condensed phase. Time-resolved x-ray scattering experiments provide a window for observing the structural dynamics that occur during certain physical transformations. This is achieved by using femtosecond (fs) x-ray pulses to monitor laser initiated dynamics, selectively track the time-dependent evolution of nonequilibrium atomic structures, and extract the shape of a photoinduced PES [1-4].

This approach has proven crucial to investigating the influence of carrier excitation on the stability of tetrahedrally bonded semiconductors. Theoretical, experimental, and simulation studies of these systems indicate that extreme carrier densities destabilize the crystal structure and
PACS numbers: 63.20.Kr, 61.10.-i, 64.70.Dv, 78.47.+p

lead to nonthermal melting [3,5-17]. Theoretical studies predict a rapid reduction in the shear restoring force when the excited carrier density exceeds a few percent of the valence band electron density [5-7]. A further doubling of the carrier density eliminates the shear restoring force, transforms the room temperature potential energy minimum into a saddle point, and leads to accelerated atomic disordering.

The initial ultrafast $\mathrm{x}$-ray diffraction studies of laserexcited InSb at the Sub-Picosecond Pulse Source (SPPS) determined that inertial atomic displacements on a lasersoftened potential energy surface dominate the response to intense optical excitation during the first 500 fs for a range of laser fluences [3]. This predominance of inertial dynamics in a wide fluence range had not been predicted by either theory or simulation. While the studies of Lindenberg et al. [3] and Gaffney et al. [15] covered the mean carrier density range over which theory predicted crystal stability to rapidly change, they did not observe a rapid onset of lattice softening or evidence of accelerated crystal disordering. By quadrupling the carrier density range previously investigated, the present study has confirmed the rapid onset of lattice softening as a function of carrier density and the appearance of accelerated crystal disordering. 
The $\mathrm{x}$ rays at SPPS are produced using compressed electron bunches accelerated by the SLAC linear accelerator which results in $2 \times 10^{6}$ photons in an $80 \mathrm{fs}$ FWHM pulse at $8.9 \mathrm{keV}$ with a $1.5 \%$ bandwidth into a 200 by $400 \mu \mathrm{m}$ spot [18]. The laser pulse used to pump the sample starts in a Ti:sapphire oscillator phase locked to a submultiple of the rf driving the linac. The laser output is centered at $800 \mathrm{~nm}$ and goes through two stages of amplification, resulting in $20 \mathrm{~mJ} /$ pulse in $50 \mathrm{fs}$ at the sample, which when convolved with the x-ray pulse yields a Gaussian cross correlation of 100 fs FWHM. The instrument response is $180 \mathrm{fs}$ FWHM once the $\mathrm{x}$-ray image resolution is taken into consideration. Experiments were performed using a noncollinear geometry, as has been described previously $[3,15]$ in order to overcome the instrinsic jitter in electron bunch time of arrival [19]. An InSb wafer asymmetrically cut at $25^{\circ}$ with respect to the (111) vector allowed the $\mathrm{x}$ rays to be at grazing incidence with the laser incident at an angle of $25^{\circ}$. The noncollinear geometry creates a difference in time of arrival between the laser pump and $\mathrm{x}$-ray probe, thereby mapping the time axis onto the spatial extent of the crystal. With the specific angles of incidence used, the length of the x-ray spot across the InSb wafer corresponds to a time sweep of $\sim 8 \mathrm{ps}$.

In order to study a broad range of mean carrier densities, we varied the laser fluence from 37 to $180 \mathrm{~mJ} \mathrm{~cm}^{-2}$ and probed the excited crystal with two different x-ray incidence angles. At $0.4^{\circ}$ incidence angle, $95 \%$ of the diffracted $\mathrm{x}$ rays probe a depth of $\sim 140 \mathrm{~nm}$, whereas $0.3^{\circ}$ incidence reduces the penetration depth to $\sim 50 \mathrm{~nm}$, thereby probing a more highly excited region of the sample [20]. Regardless of the specific angle used, the measured diffraction signal represents an average over the energy deposition profile, which is a function of the optical penetration depth. Hence, the $0.4^{\circ}$ probe angle measures the signal from the region probed at $0.3^{\circ}$ as well as signal from deeper penetration, leading to a lower mean carrier density.

Prior investigations by Lindenberg et al. and Gaffney et al. have demonstrated that the time-dependent decay of the diffraction intensity results from a time-dependent mean-square displacement (msd), $\left\langle u^{2}(t)\right\rangle$, within a timedependent Debye-Waller model $[3,15]$. This model gives a time-dependent diffraction intensity, $I(Q, t)$, equal to

$$
I(Q, t)=\exp \left(-\frac{Q^{2}\left\langle u^{2}(t)\right\rangle}{3}\right)
$$

where $Q$ is the Bragg peak reciprocal lattice vector. We have used the Debye model for the crystal vibrations in the high temperature limit to calculate $\left\langle u^{2}(t)\right\rangle$ in an attempt to understand the interplay of the excited state potential energy surface and the time-dependent diffraction intensity. Laser excitation is treated as a uniform softening of the Debye phonon distribution, $\alpha=\frac{\omega_{f}}{\omega_{i}}$, where $\omega_{i}$ represents the initial, unperturbed phonon frequencies and $\omega_{f}$ represents the phonon frequencies after impulsive softening. This model does not account for anharmonicities or atomic collisions and assumes the mean atomic positions remain at the equilibria positions of the zinc blende lattice, while the width of the msd increases for $\alpha^{2}<1$. In this limit,

$$
\left\langle u^{2}(t)\right\rangle=\frac{9 k_{B} T}{2 M \omega_{D}^{2}}\left[\left(1+\frac{1}{\alpha^{2}}\right)+\frac{\sin \left(2 \alpha \omega_{D} t\right)}{2 \alpha \omega_{D} t}\left(1-\frac{1}{\alpha^{2}}\right)\right],
$$

where $\omega_{D}$ is the Debye frequency. In order to model the diffracted intensity, this expression for $\left\langle u^{2}(t)\right\rangle$ is substituted into Eq. (1). The model describes the average dynamics of atoms that result from an isotropic and uniform ultrafastlaser modification of the potential energy surface.

This time-dependent Debye-Waller model provides context for understanding the experimental results presented in Fig. 1. For the range of lattice softening where inertial dynamics provide the predominant mechanism of disordering, the model delineated above reduces to a Gaussian for the first 0.5 ps. This simplification of the model has been used in fitting the signals measured with a $0.4^{\circ}$ probe as was done previously by Lindenberg et al. [3]. In order to account for atomic diffusion occurring after the onset of inertial dynamics, a normalized sum of a Gaussian and an exponential provides the best agreement with the data [15]. A Gaussian decay with a time constant of $400 \mathrm{fs}$ dominated the initial decay in prior measurements and was attributed to inertial disordering [3]. In the harmonic approximation, inertial disordering occurs at a rate dictated by the average thermal velocity, $\left\langle v^{2}\right\rangle=\frac{3 k_{B} T}{M}$, which results in $\left\langle u^{2}(t)\right\rangle=$ $\frac{3 k_{B} T t^{2}}{M}$ and a room temperature Gaussian time constant of $\tau=\sqrt{\frac{M}{Q^{2} k_{B} T}}=400 \mathrm{fs}$, where $M$ is the average mass of In and $\mathrm{Sb}$.

With an x-ray incidence angle of $0.4^{\circ}$ and laser fluence of $37 \mathrm{~mJ} \mathrm{~cm}^{-2}$, the signal decays with an exponential time constant of $5 \pm 0.5$ ps and no Gaussian component. A $20 \%$ increase in fluence to $44 \mathrm{~mJ} \mathrm{~cm}{ }^{-2}$ results in a Gaussian decay with a $450 \pm 50 \mathrm{fs}$ time constant. For all laser fluences of $44 \mathrm{~mJ} \mathrm{~cm}^{-2}$ or more probed at $0.4^{\circ}$, the Gaussian time constant is within the $95 \%$ confidence limit of the 400 fs decay predicted for inertial dynamics. The data at lowest fluence shows a strong deviation from the trend given by the other data points which all show significant Gaussian components. This deviation reflects the apparent absence of an inertial component in the disordering, as shown in Fig. 1(a). The observation of the decay in diffracted intensity with and without inertial contributions over a narrow laser fluence range provides direct evidence for a sharp onset of lattice softening as a function of carrier density, similar to that predicted by theory. In the absence of lattice softening, decay in the diffraction intensity is attributed to a rise in the msd following the transfer of energy from the electrons to the lattice vibrations, with an expected rate of a few ps [21].

In order to probe an even higher carrier density regime, the $x$-ray incidence angle was reduced to $0.3^{\circ}$ while exciting with a fluence of $160 \mathrm{~mJ} \mathrm{~cm}^{-2}$. The resulting signal shows dynamics significantly faster than the predomi- 


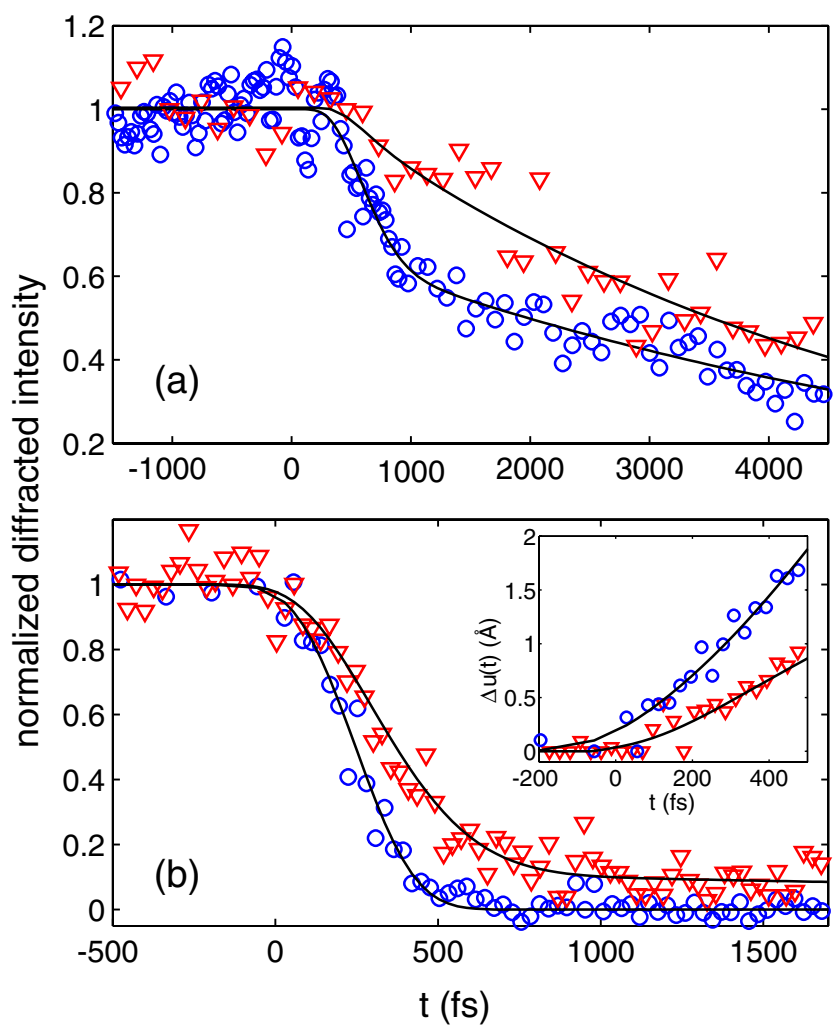

FIG. 1 (color online). Normalized time-dependent diffracted intensity for the (111) Bragg peak with corresponding fits. (a) Observation of the carrier density threshold for the onset of inertial atomic motion with $37 \mathrm{~mJ} \mathrm{~cm}^{-2}(\nabla)$ and $44 \mathrm{~mJ} \mathrm{~cm}^{-2}$ (O) at $0.4^{\circ} \mathrm{x}$-ray angle of incidence. (b) Observation of predominantly inertial dynamics for $0.4^{\circ} \mathrm{x}$-ray angle of incidence and $180 \mathrm{~mJ} \mathrm{~cm}^{-2}$ laser fluence $(\nabla)$ and accelerated atomic disordering with $0.3^{\circ} \mathrm{x}$-ray angle of incidence and $160 \mathrm{~mJ} \mathrm{~cm}^{-2}$ laser fluence $(O)$. The $0.4^{\circ}$ data are shown with a fits to the normalized sum of a Gaussian and exponential, while the $0.3^{\circ}$ data is shown with a fit to the accelerated dynamics model, both of which are described in the text. Inset displays the msd for the data and fits as extracted from Eq. (1) as $\Delta u(t)=\sqrt{-\frac{3 \ln [I(t)]}{Q^{2}}}$.

nantly inertial dynamics observed with a $0.4^{\circ} \mathrm{x}$-ray incidence angle at comparable and higher fluence. Because of the deviation from inertial dynamics, the full model as detailed in Eq. (2) is used in fitting this data. Within this model, $\alpha \omega_{D}$ determines the time-dependent crystal dynamics. For a real, positive $\alpha \omega_{D}$, the model results in a squeezed state with inertial disordering for early times. For $\alpha \omega_{D}=0$, the model produces inertial dynamics at all time delays, while for an imaginary $\alpha \omega_{D}$, the model predicts accelerated disordering. Fitting the $0.3^{\circ}$ data with this model results in a carrier-induced lattice destabilization characterized by $\alpha \omega_{D}=0.6 i \pm 0.1 i \mathrm{THz}$ which suggests the existence of a carrier generated inversion of the potential energy surface. Given the prior observation of anisotropy in the atomic dynamics [15], the onsets for carrierinduced softening and accelerated disordering observed in the $\langle 111\rangle$ direction need not be the same in other crystallographic directions.
Figure 2 displays the x-ray diffraction decay dynamics for three different cases of lattice softening as predicted by this model. The inset shows the predicted time for the diffraction intensity to drop to $1 / e$ for this model as a function of $\alpha \omega_{D}$. Disordering dynamics appear inertial in time for a range of crystal softening during the first $0.5 \mathrm{ps}$ or more. For time delays much longer that $0.5 \mathrm{ps}$, atomic collisions and the onset of diffusion will cause the observed dynamics to deviate significantly from those predicted for a softened, harmonic crystal, limiting the applicability of this model to the early time dynamics.

In order to compare the experimental fluence dependence of the disordering rate to theory and simulation, the carrier density must be estimated. A variety of factors influence the time-dependent carrier density profile, including the linear and nonlinear absorption cross-sections, ambipolar diffusion, Auger recombination, and impact ionization. Since the rate of these processes are not known in the presence of a dense electron-hole plasma, only a coarse estimate of the carrier density is possible. The parameters of Rousse et al. have been used to determine the laser absorption profile [14]. This profile is then used to calculate the average deposited laser energy density between the surface and the depth at which the x-ray intensity has been attenuated to $5 \%$ of the incident intensity. An approximation to the calculated InSb density of states is utilized to determine the carrier temperature and density needed to support the average absorbed laser energy density within the $\mathrm{x}$-ray probing depth [22,23]. The average absorbed laser energy density is a function of excitation fluence and probe depth. This model is consistent with the expected fast electronic equilibration [24] and compara-

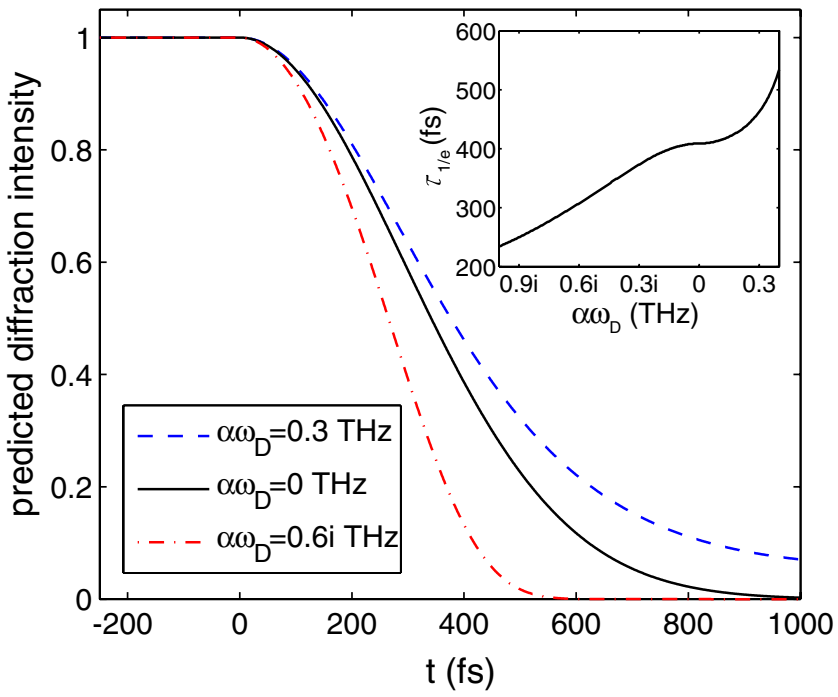

FIG. 2 (color online). Plot of the predicted time-dependent diffraction intensity as given by the model described in the text for $\alpha \omega_{D}=0.3 \mathrm{THz}, \quad \alpha \omega_{D}=0 \mathrm{THz}, \quad$ and $\alpha \omega_{D}=$ $0.6 i \mathrm{THz}$. Predicted diffraction intensity has oscillatory character for positive softening on longer time scales. Inset displays $\tau_{1 / e}$ as a function of $\alpha \omega_{D}$. 


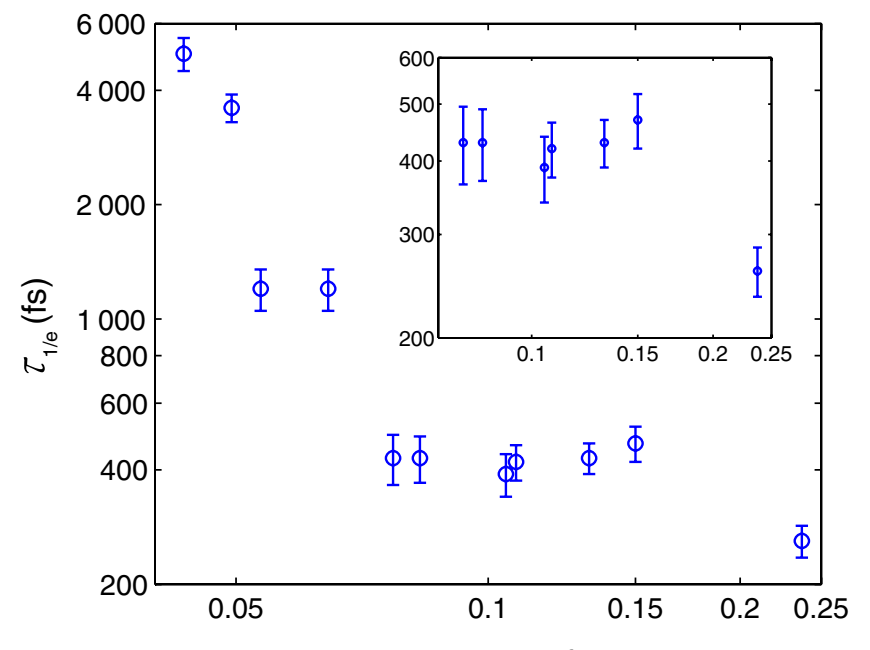

estimated carrier fraction

FIG. 3 (color online). Log plot of $\tau_{1 / e}$ as a function of carrier density. Inset displays the high carrier density regime. See text for the definition of $\tau_{1 / e}$ and method of estimation for the carrier density.

tively slow rate of phonon emission [21] and ambipolar diffusion in a dense plasma [25].

In order to compare disordering time scales, the time required for the diffraction intensity to drop to $1 / e$ of its original value $\left(\tau_{1 / e}\right)$ is used. Figure 3 presents the $\tau_{1 / e}$ times for various carrier densities as extracted from fits to the data using the phenomenological sum of a Gaussian and exponential. Below $\sim 5 \%$ excited carriers, electronic relaxation via phonon emission is the cause of lattice disordering, rather than direct modification of the potential energy surface. As more carriers are excited from the valence to conduction band, an inertial contribution dominates on early time scales, resulting from motion on a lasersoftened potential energy surface. For a carrier density above $\sim 20 \%$, disordering occurs too fast to be accounted for by inertial motion, consistent with a carrier driven lattice instability and a repulsive potential. The abrupt onset of potential softening and the appearance of a repulsive potential energy surface at high carrier densities had been predicted by theory [5-7], but had not been confirmed by prior experiment $[3,10,11,15]$. The inertial dynamics observed for a wide range of carrier density can be qualitatively accounted for with a classical Debye model where the crystal dynamics originate from a uniform softening of the phonon distribution. The crystal appears to soften slowly with carrier density following the rapid carrier dependent onset of softening, a conclusion in contrast with the predictions of theory and simulation. This analysis provides a means of extracting the shape of the potential energy surface from the time-dependent diffraction signal. These results further demonstrate the utility of femtosecond x-ray diffraction for characterizing excited state potential energy surfaces and ultrafast atomic dynamics.

Portions of this research were supported by the U.S. Department of Energy, Office of Basic Energy Science through direct support for the SPPS, as well as individual investigators and SSRL, a national user facility operated by Stanford University. Additional support for the construction of SPPS was provided in part by Uppsala University and the Swedish Research Council. Portions of this work were supported by the W.M. Keck Foundation, Stanford PULSE Center, and University of Michigan FOCUS Center. K. S. T. and D. V.D.L. gratefully acknowledge financial support by the Deutsche Forschungsgemeinschaft. J.L., K.S.T., and D. V.D.L. acknowledge the support of the European Commission through the FEMTO, X-RAY FEL PUMP-PROBE, and XPOSE projects. J. L. acknowledges support from the Wallenberg Research Link and The Swedish Foundation for Strategic Research. D. A. R. acknowledges critical conversations with S. Fahy and R. Merlin on the effects of phonon squeezing.

*Electronic address: kgaffney@slac.stanford.edu

[1] K. Sokolowski-Tinten et al., Nature (London) 422, 287 (2003).

[2] A. Cavalleri et al., Nature (London) 442, 664 (2006).

[3] A. M. Lindenberg et al., Science 308, 392 (2005).

[4] D. M. Fritz et al., Science 315, 633 (2007).

[5] R. Biswas and V. Ambegaokar, Phys. Rev. B 26, 1980 (1982).

[6] P. Stampfli and K. H. Bennemann, Phys. Rev. B. 42, 7163 (1990).

[7] P. Stampfli and K. H. Bennemann, Phys. Rev. B. 46, 10686 (1992).

[8] V. Recoules et al., Phys. Rev. Lett. 96, 055503 (2006).

[9] T. Dumitrica, A. Burzo, Y. Dou, and R. E. Allen, Phys. Status Solidi B 241, 2331 (2004).

[10] K. Sokolowski-Tinten, J. Bialkowski, and D. von der Linde, Phys. Rev. B. 51, 14186 (1995).

[11] S. V. Govorkov, T. Schroder, I. L. Shumay, and P. Heist, Phys. Rev. B. 46, 6864 (1992).

[12] I. L. Shumay and U. Hofer, Phys. Rev. B. 53, 15878 (1996).

[13] L. Huang, J. P. Callan, E. N. Glezer, and E. Mazur, Phys. Rev. Lett. 80, 185 (1998).

[14] A. Rousse et al., Nature (London) 410, 65 (2001).

[15] K. J. Gaffney et al., Phys. Rev. Lett. 95, 125701 (2005).

[16] K. Sokolowski-Tinten et al., Phys. Rev. Lett. 87, 225701 (2001).

[17] T. Dumitrica and R.E. Allen, Phys. Rev. B. 66, 081202 (2002).

[18] P. Krejcik et al., in Proceedings of the 2003 Particle Accelerator Conference, edited by J. Chew, S. Lucas, and S. Webber (IEEE, Piscataway, NJ, IEEE, Portland, OR, 2003), p. 423.

[19] A. L. Cavalieri et al., Phys. Rev. Lett. 94, 114801 (2005).

[20] B. L. Henke, E. M. Gullikson, and J. C. Davis, At. Data Nucl. Data Tables 54, 181 (1993).

[21] R. F. Leheny et al., Solid State Commun. 31, 809 (1979).

[22] J. R. Chelikowsky and M. L. Cohen, Phys. Rev. B. 14, 556 (1976).

[23] M. Combescot and J. Bok, J. Lumin. 30, 1 (1985).

[24] W. H. Knox et al., Phys. Rev. Lett. 61, 1290 (1988).

[25] E. J. Yoffa, Phys. Rev. B. 21, 2415 (1980). 